\title{
The Chemical Evolution of Omega Centauri
}

\author{
Donatella Romano \\ INAF-Osservatorio Astronomico di Bologna, Via Ranzani 1, I-40127, Bologna, Italy \\ email: donatella.romano@oabo.inaf.it
}

\begin{abstract}
The globular cluster $\omega$ Cen is an outstanding object in terms of both its chemical and kinematic properties. Its large mass, spread in element abundances, chemical and kinematical segregations, as well as its peculiar orbit, all suggest that it is the surviving remnant of a larger system. In this contribution we deal with the chemical evolution of $\omega$ Cen in the framework of a model where it is the remnant of a dwarf spheroidal galaxy evolved in isolation and then swallowed and partially disrupted by the Milky Way. Both infall of primordial matter and metal-enriched gas outflows are necessary in order to reproduce the observed stellar metallicity distribution function, age-metallicity relation and several abundance ratios. Yet, as long as an ordinary stellar initial mass function and standard stellar yields are adopted, we fail by far to get the enormous helium enhancement required to explain the double main sequence of $\omega$ Cen.
\end{abstract}

Keywords. Galaxies: dwarf, galaxies: evolution, globular cluster: individual ( $\omega$ Centauri)

\section{Chemical enrichment in $\omega$ Cen's progenitor}

It has been suggested that $\omega$ Cen, the most massive globular cluster of the Milky Way, might be the surviving remnant of a dwarf Galactic satellite evolved in isolation and then accreted by the Milky Way a long time ago. We find that, in the framework of such a scenario, its main chemical properties can be nicely reproduced by a chemical evolution model which allows for both infall and outflows from the parent system (see Romano et al. 2007 for basic assumptions and equations).

In Fig. 1 we show our predictions (solid lines) for the stellar metallicity distribution function (MDF, left panel), age-metallicity relation (AMR, upper middle panel), helium enhancement history (lower middle panel) as well as several alpha-to-iron abundance ratios as functions of $[\mathrm{Fe} / \mathrm{H}]$ (right panels). Theoretical expectations are compared to observed quantities (see figure caption for references). In our model, the star formation lasts 3 Gyr (see Stanford et al. 2006, and references therein) and the stars distribute over the 0.1-100 $\mathrm{M}_{\odot}$ mass range according to an extrapolated Salpeter (1955) IMF. In order to reproduce the observed MDF and AMR, we must assume that the parent galaxy experienced an early infall of primordial gas and that $S N$ ejecta were selectively removed through galactic winds. A similar scenario was proposed also by Ikuta \& Arimoto (2000), who however assumed a relatively short-lasting star formation history $(\sim 0.3 \mathrm{Gyr})$, a scenario no longer supported by the observations (e.g. Hilker et al. 2004; Stanford et al. 2006).

While the behaviour of several $\alpha$-elements as a function of metallicity is fairly well reproduced in the framework of our models (Fig. 1, right panels), the predicted flat behaviour of the He enhancement versus $[\mathrm{Fe} / \mathrm{H}]$ contrasts sharply with the large helium excess implied by blue main sequence (bMS) observations (Fig. 1, lower middle panel; the box represents the level of helium enhancement required for bMS stars according to Norris 2004 and Piotto et al. 2005). By adopting a 'standard' IMF, our chemical evolution model fails to get the enormous He enrichment required to explain the bMS data independently of the choice of stellar yields (see Romano et al. 2007 for details). Yet, 

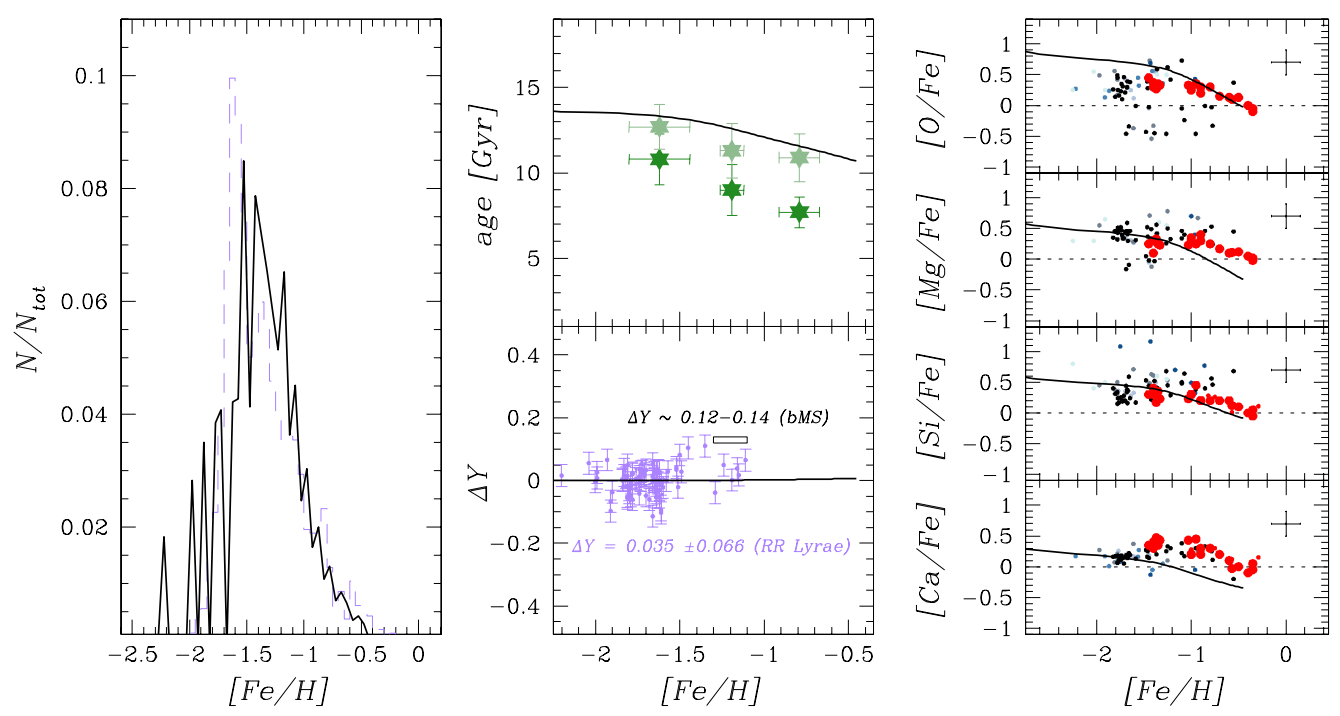

Figure 1. Left panel. Predicted MDF for $\omega$ Cen's stars (solid curve) compared to the observed one by Sollima et al. (2005; dashed histogram). Middle panels. Top: Predicted AMR (solid curve) versus observations (stars) for two different assumptions of distance modulus and reddening (see Hilker et al. 2004). Bottom: Helium enrichment in $\omega$ Cen: theory (solid curve) versus 'observations' (bMS stars, box; Norris 2004; Piotto et al. 2005 and RR Lyræ data, bullets; Sollima et al. 2006) Right panels. Predicted $[\alpha / \mathrm{Fe}]$ vs $[\mathrm{Fe} / \mathrm{H}]$ behaviour for several $\alpha$-elements (solid lines) compared to observational data obtained from both high-resolution optical (dots) and low-resolution infrared (big filled circles) spectra. Literature values have been homogenized (see Romano et al. 2007 for the adopted criteria).

the low He abundance we find in the course of the whole $\omega$ Cen evolution is consistent with recent RR Lyræ data by Sollima et al. (2006; Fig. 1, lower middle panel, dots), which seem to suggest that two distinct populations with the same metallicity but very different helium content might inhabit the cluster.

\section{Acknowledgements}

The results presented here have been obtained in collaboration with F. Matteucci, M. Tosi, E. Pancino, M. Bellazzini, F. R. Ferraro, M. Limongi and A. Sollima. I warmly thank F. Matteucci and M. Tosi for enlightening discussions on the chemical evolution of galaxies in the course of the years.

\section{References}

Hilker, M., Kayser, A., Richtler, T., \& Willemsen, P. 2004, A\&A 422, L9

Ikuta, C. \& Arimoto, N. 2000, A\& $A$ 358, 535

Norris, J. E. 2004, ApJ 612, L25

Piotto, G., et al. 2005, ApJ 621, 777

Romano, D., Matteucci, F., Tosi, M., Pancino, E., Bellazzini, M., Ferraro, F.R., Limongi, M., \& Sollima, A. 2007, MNRAS in press (astro-ph/0701162)

Salpeter, E. E. 1955, ApJ 121, 161

Sollima, A., Ferraro, F. R., Pancino, E., \& Bellazzini, M. 2005, MNRAS 357, 265

Sollima, A., Borissova, J., Catelan, M., Smith, H. A., Minniti, D., Cacciari, C., \& Ferraro, F. R. 2006, ApJ 640, L43

Stanford, L. M., Da Costa, G. S., Norris, J. E., \& Cannon, R. D. 2006, ApJ 647, 1075 